\title{
Left and right ventricle assessment with Cardiac CT: validation study vs. Cardiac MR
}

\author{
Erica Maffei • Giancarlo Messalli • Chiara Martini • \\ Koen Nieman • Onofrio Catalano • Alexia Rossi • \\ Sara Seitun • Andrea I Guaricci • Carlo Tedeschi • \\ Nico R. Mollet • Filippo Cademartiri
}

Received: 23 April 2011 /Revised: 5 October 2011 / Accepted: 8 October 2011 / Published online: 24 January 2012

(C) The Author(s) 2012. This article is published with open access at Springerlink.com

\begin{abstract}
Objectives To compare Magnetic Resonance (MR) and Computed Tomography (CT) for the assessment of left (LV) and right (RV) ventricular functional parameters.

Methods Seventy nine patients underwent both Cardiac CT and Cardiac MR. Images were acquired using short axis (SAX) reconstructions for CT and 2D cine b-SSFP (balanced-steady state free precession) SAX sequence for MR, and evaluated using dedicated software.

Results CT and MR images showed good agreement: LV EF (Ejection Fraction) $(52 \pm 14 \%$ for CT vs. $52 \pm 14 \%$ for MR; $r=$ $0.73 ; p>0.05$ ); RV EF ( $47 \pm 12 \%$ for CT vs. $47 \pm 12 \%$ for MR;
\end{abstract}

Electronic supplementary material The online version of this article (doi:10.1007/s00330-011-2345-6) contains supplementary material, which is available to authorized users.

E. Maffei $\cdot$ C. Martini $\cdot$ S. Seitun $\cdot$ F. Cademartiri $(\bowtie)$

Cardiovascular Radiology Unit, Giovanni XXIII Hospital,

31050 Monastier di Treviso, Italy

e-mail: filippocademartiri@gmail.com

G. Messalli $\cdot$ O. Catalano

SDN Foundation - IRCCS,

Naples, Italy

C. Martini - K. Nieman · A. Rossi • N. R. Mollet • F. Cademartiri Department of Radiology and Cardiology,

Erasmus Medical Center,

Rotterdam, The Netherlands

A. I. Guaricci

Department of Cardiology,

Azienda Ospedaliero-Universitaria di Foggia,

Foggia, Italy

C. Tedeschi

Department of Cardiology, Ospedale San Gennaro,

Naples, Italy $r=0.74 ; p>0.05)$; LV EDV (End Diastolic Volume) $(74 \pm$ $21 \mathrm{ml} / \mathrm{m}^{2}$ for CT vs. $76 \pm 25 \mathrm{ml} / \mathrm{m}^{2}$ for MR; $r=0.59 ; p>0.05$ ); RV EDV $\left(84 \pm 25 \mathrm{ml} / \mathrm{m}^{2}\right.$ for CT vs. $80 \pm 23 \mathrm{ml} / \mathrm{m}^{2}$ for MR; $r=$ $0.58 ; p>0.05)$; LV ESV (End Systolic Volume)(37 $\pm 19 \mathrm{ml} / \mathrm{m}^{2}$ for CT vs. $38 \pm 23 \mathrm{ml} / \mathrm{m}^{2}$ for MR; $\left.r=0.76 ; p>0.05\right)$; RV ESV $\left(46 \pm 21 \mathrm{ml} / \mathrm{m}^{2}\right.$ for CT vs. $43 \pm 18 \mathrm{ml} / \mathrm{m}^{2}$ for MR; $r=0.70 ; p>$ 0.05 ). Intra- and inter-observer variability were good, and the performance of CT was maintained for different EF subgroups. Conclusions Cardiac CT provides accurate and reproducible LV and RV volume parameters compared with MR, and can be considered as a reliable alternative for patients who are not suitable to undergo MR.

Key Points

- Cardiac-CT is able to provide Left and Right Ventricular function.

- Cardiac-CT is accurate as MR for LV and RV volume assessment.

- Cardiac-CT can provide accurate evaluation of coronary arteries and $L V$ and $R V$ function.

Keywords Cardiac magnetic resonance - Cardiac computed tomography $\cdot$ Left ventricle assessment $\cdot$ Right ventricle assessment $\cdot$ Ejection fraction

\section{Introduction}

Correct and reproducible evaluation of left (LV) and right ventricular (RV) functional parameters underly appropriate decision making in cardiac and pulmonary diseases [1-7]. Owing to high temporal, spatial and contrast resolution the Magnetic Resonance (MR) 2D-b-SSFP (balanced-Steady State Free Precession) sequence guarantees the excellent results in terms of accuracy of LV and RV volume assessment and is nowadays regarded as the in vivo reference 
standard for this purpose [8-13]. Recently, as a result of improved temporal resolution, numerous studies have explored the role of cardiac $\mathrm{CT}$ in providing $\mathrm{LV}$ and $\mathrm{RV}$ functional parameters [14-32]; in fact it is important to have alternative tools to evaluate an important parameter, such as for example EF, when other techniques cannot be used. CT has some drawbacks in respect of Echo and MRI, such as radiation dose and contrast medium administration, but it is important to demonstrate that in particular situations, like in patients with poor echocardiographic compliance, and contra-indications to MR, CT can offer a reliable alternative to assess ventricular function in a fast way and with contemporary information on the coronary artery tree status.

The importance of an alternative functional imaging investigation is even more evident for the RV, which has a complex shape that lends itself poorly for echocardiographic functional assessment. Our results suggest that CT could be a reliable second option to MR, for instance in patients with (corrected) grown-up congenital heart disease.

The majority of studies explored singularly the possibility of performing $\mathrm{LV}$ and $\mathrm{RV}$ analysis with $\mathrm{CT}$ and so far the feasibility and reproducibility of concurrent left and right ventricular volume assessment by $\mathrm{CT}$ has not been compared with MR in a large sample size. We think it is really important to test the accuracy of contemporary LV and RV analysis because LV abnormalities can frequently involve RV function and vice versa and because concurrent assessment can speed up the acquisition of important information especially when there is no time to lose.

We report our experience in 79 patients who underwent CT for evaluation of coronary artery disease.

\section{Material and methods}

\section{Patients}

Between April 2007 and October 2009 we prospectively enrolled 100 patients who underwent CT for evaluation of coronary artery disease. For bi-ventricular volume assessment, patients were also scheduled for an MR within one week after CT. We adhered to the exclusion criteria reported in literature [33-35]: for CT, we excluded patients with a) an heart rate $>65 \mathrm{bpm}$ (beats per minute) not responding to betablockers; b) atrial fibrillation and concomitant high ventricular response; c) known reactions to contrast medium; d) renal insufficiency (creatinine $>1.5 \mathrm{mg} / \mathrm{dL}$ ); e) impaired pulmonary function (unable to perform a 12-s breath-hold); for MR, we excluded patients with a) claustrophobia; b) pacemaker/other non MR compatible devices [34]. Twenty-one patients were excluded because of unsuitability to undergo MR (11 for claustrophobia; 3 for increasing dyspnoea during acquisition; 4 for ICD; 2 for pacemaker; 1 for a Starr-Edwards mitral valve); within our cohort no patient needed exclusion from CT. Complete CT and MR datasets could be acquired in 79 patients (mean age: 58; 46 male) (Table 1).

Written informed consent was obtained from all patients, and the study was approved by the Ethics Review Board of our hospital.

\section{CT protocol}

Upon arrival, blood pressure and heart rate (HR) were measured and 5-20 mg of intra-venous atenolol were administered if the HR exceeded $65 \mathrm{bpm}$, in order to reach a HR below $65 \mathrm{bpm}$. Twenty-five patients required treatment with $\beta$ blockers before $\mathrm{CT}$; the others had no necessity (40 patients) or had contraindications (14 patients). ECG-gated 64-slice spiral CT (Sensation 64, Siemens, Forchheim, Germany) was performed without ECG-triggered tube modulation: detector collimation: $32 \times 0.6 \mathrm{~mm}$, Z-axis focal spot alternation resulting in simultaneous acquisition of 64 slices; gantry rotation: $330 \mathrm{~ms}$; effective temporal resolution $165 \mathrm{~ms}$; table feed per rotation: $3.84 \mathrm{~mm}$; tube voltage $120 \mathrm{kVp}$; tube current 800-950 mAs; direction in which data acquisition proceeded : cranio-caudal. Just before the $\mathrm{CT}$ data acquisition $0.3 \mathrm{mg}$ of sublingual nitroglycerin was administered in the absence of contraindications. For contrast enhancement, $110 \mathrm{~mL}$ of contrast medium (Iomeprol, Iomeron 400, Bracco, Milan, Italy) was administered intravenously at $5 \mathrm{~mL} / \mathrm{s}(2 \mathrm{~g}$ iodine/s) flow rate, followed by $40 \mathrm{~mL}$ of saline chaser at same flow rate [17]. A bolus tracking technique for contrast bolus arrival and data acquisition synchronisation was used. The anatomical coverage extended from the tracheal bifurcation to the diaphragm. In patients who previously underwent bypass graft surgery, the start was positioned just at clavicle level to include internal mammary artery origin. Using a partial reconstruction algorithm (requiring $180^{\circ}$ of projections) thinslice images were reconstructed for the coronary evaluation. In addition functional MPR (multiplanar reconstruction) images were reconstructed using dedicated CT software (Syngo CT-2007A; Siemens, Forchheim, Germany). Using the standard cardiac planes for orientation, 8-mm MPR thick with a 2-mm gap images of the ventricles, extending from the base to the apex, were reconstructed throughout the cardiac cycle at $5 \%$ intervals (Fig. 1).

\section{MR protocol}

MR was performed at $1.5 \mathrm{~T}$ (Achieva, Philips Medical Systems, Best, The Netherlands) with the following acquisition parameters: maximum gradient strength of $66 \mathrm{mT} / \mathrm{m}$, maximum slew rate $180 \mathrm{mT} / \mathrm{m} \times \mathrm{ms}$, maximum gradient strength during Cine-Cardiac MR acquisition $33 \mathrm{mT} / \mathrm{m}$, and maximum slew rate during Cine-Cardiac MR acquisition $180 \mathrm{mT} / \mathrm{m} \times \mathrm{ms}$. Five-element synergy cardiac coil 
Table 1 Demographics

\begin{tabular}{ll}
\hline Population & Total \\
\hline Number & 79 \\
Age (mean \pm SD; median; range) & $58 \pm 17(58 ; 24-89)$ \\
Male/Female & $46 / 33$ \\
Cardiovascular Risk Factors & \\
Hypertension (\%) & $35(44.3)$ \\
Dyslipidaemia (\%) & $15(18.9)$ \\
Diabetes mellitus (\%) & $12(15.2)$ \\
Nicotine abuse (\%) & $23(29.1)$ \\
Family history of cardiovascular disease (\%) & $18(22.8)$ \\
BSA (m ${ }^{2}$; mean \pm SD) & $1.9 \pm 0.2$
\end{tabular}

The Table shows demographics of the study population

Abbreviations: $S D$ Standard Deviation, BSA Body Surface Area (according to Mosteller's formula)

and vector electrocardiography were used for signal detection and cardiac gating. One experienced operator (4 years' Cardiac MR) performed all examinations. After initial scout imaging and reference acquisition, shortaxis cine images covering the entire left and right ventricles from base to apex were acquired during repeated end-expiratory breath holds using a 2D b-SSFP (2Dcine) sequence (Fig. 1). All acquisitions were performed holding the breath at end expiration to avoid slice misalignment [36, 37]. The imaging parameters for 2D-cine were as follows: TR (repertition time) $3.1 \mathrm{~ms}$; TE (echo time)
$1.53 \mathrm{~ms}$; flip angle $60^{\circ}$; bandwidth $1249.7 \mathrm{HZ} /$ pixel; in plane resolution $2 \times 2.3 \mathrm{~mm}$; slice thickness $8 \mathrm{~mm}$; slice gap $2 \mathrm{~mm}$; temporal resolution $32 \pm 6 \mathrm{~ms}$ (depending on heart rate); cardiac phase 30; SENSE: off; half scan: Yes.

\section{Data analysis}

A total of 158 short axis datasets, 79 obtained by MR acquisition and 79 by CT multiphasic reconstruction, were transferred to a dedicated workstation (Syngo MMWP Siemens, Forchheim, Germany) equipped with ARGUS Va60c analysis software (ARGUS, Va60c, Siemens, Forchheim, Germany), which is able to process DICOM images from images obtained using different techniques. One experienced observer ( $>5$ years in Cardiac MR and CT) blindly and randomly analyzed all MR and CT images to measure the end-diastolic volume (EDV) and end-systolic volume (ESV), and calculate the stroke volume (SV) and ejection fraction (EF) of left and right ventricle, as well as the end-diastolic wall mass (ED Wall Mass) on the left ventricle. [38]. Images acquired at the time of the R-wave of the ECG were considered to represent end-diastole (ED), while images showing the smallest detectable left ventricular cavity were considered as end-systolic (ES) [18]. Endocardial and epicardial contours were manually traced on the end-diastolic SAX images. Endocardial borders were automatically "propagated" on endsystolic phase images, and manually corrected when deemed necessary. Papillary muscles and trabeculations of the LV and

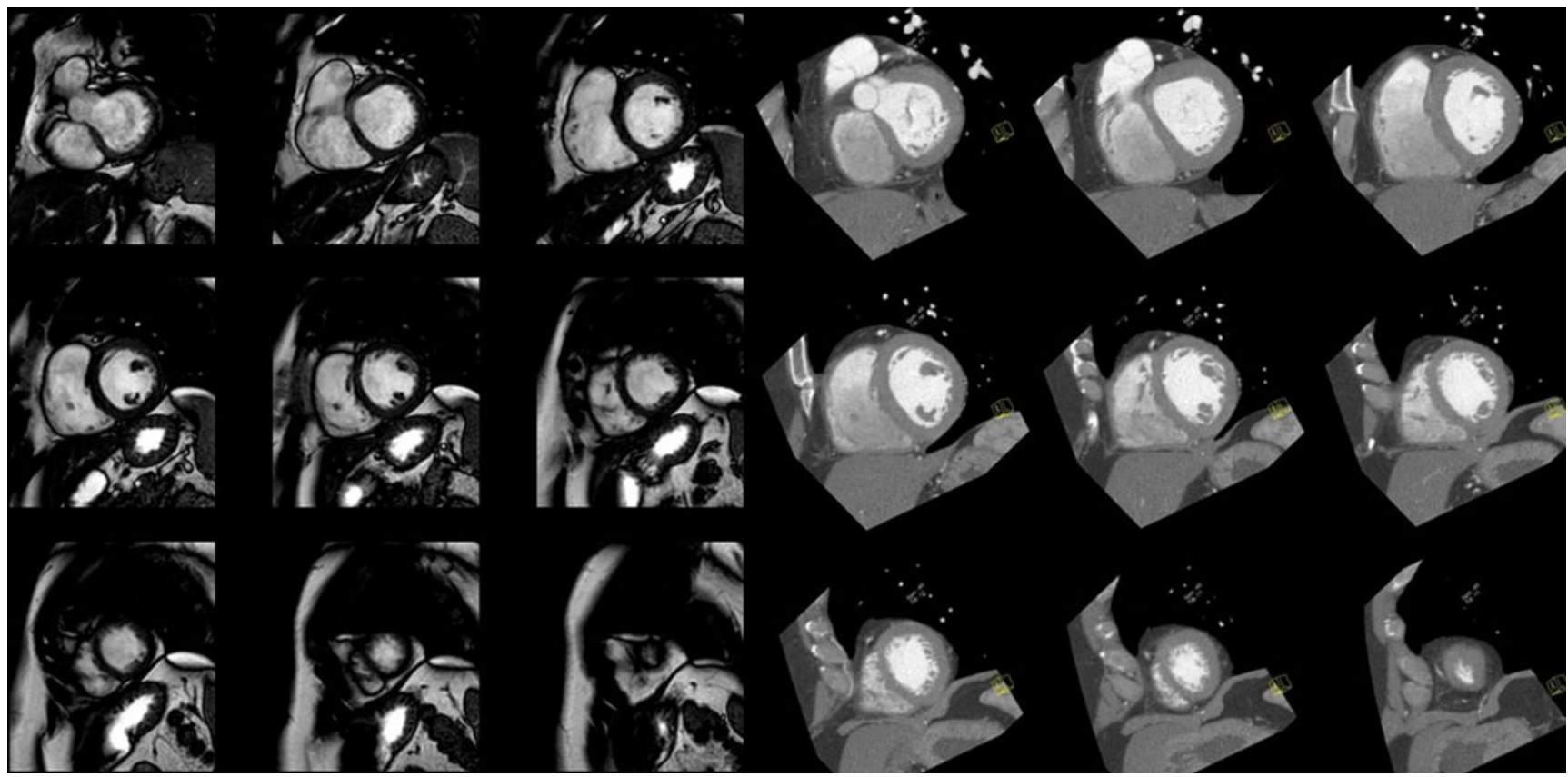

Fig. 1 Short Axis views of the Left and Right Ventricle by MR and CT. End-diastolic Short Axis views of the Left and Right Ventricle by MR and CT (MPR $8 \mathrm{~mm}$ thick reconstructions). Example of the same patient imaged with MR (on the left) and CT (on the right). Short axis views for left and right ventricular volume calculation. Abbreviations: MR Magnetic Resonance, CT Computed Tomography 
$\mathrm{RV}$ cavities were included in the LV and RV cavity volumes as previously described [39-41]. The most apical section with visible cavity was considered as apex and the most basal section with at least $50 \%$ surrounding myocardium was regarded as base [23]. EDV and ESV were calculated without geometric assumptions, using the Simpson's rule. All parameters were indexed for body surface area (BSA). For calculation of intra-observer and inter-observer variability tracing of myocardial borders was repeated after at least 1 month by the
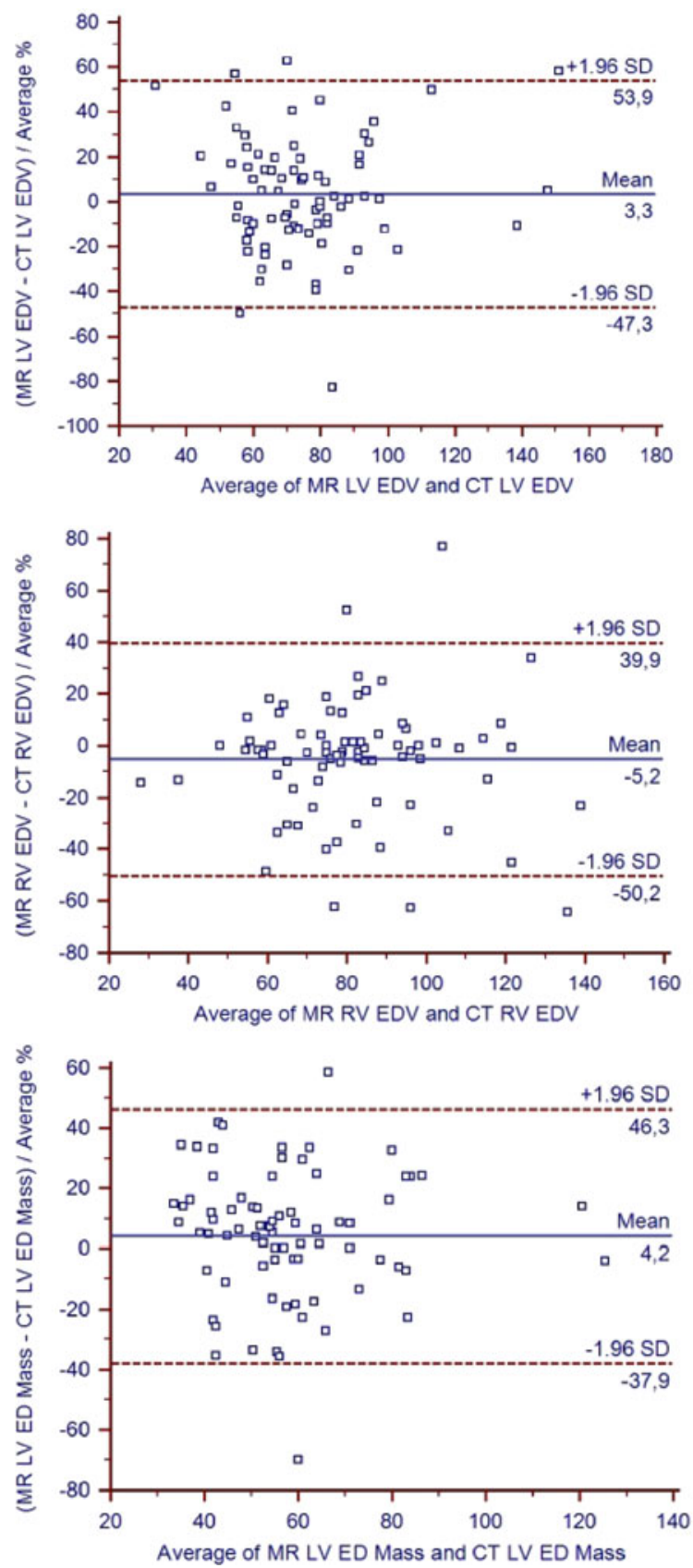

Fig. 2 Bland-Altman plots. Bland-Altman plots show good agreement for Left and Right Ventricular EF and for Left Ventricular ED wall Mass. Abbreviations: $L V$ Left Ventricle, $R V$ Right Ventricle, $E F$ same investigator, and in addition by a second investigator (4 years' Cardiac CT and Cardiac MR), who was unaware of previous results.

Statistical analysis

We hypothesized that CT can measure right and left ventricular volumes, and left myocardial mass with acceptable accuracy and reproducibility; thus we directly compared
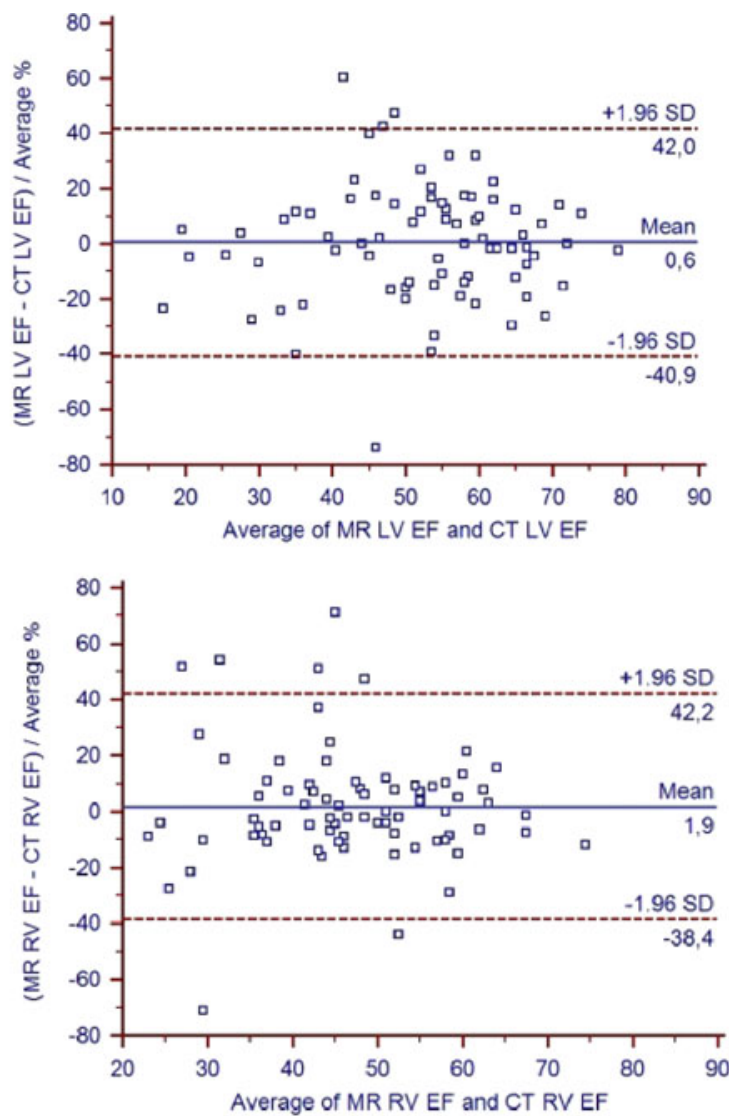

Ejection Fraction, EDV End Diastolic Volume, ED mass End Diastolic wall mass, $M R$ Magnetic Resonance, $C T$ Computed Tomography, $S D$ standard deviation 
Table 2 Ventricular function parameters

\begin{tabular}{llllll}
\hline \multicolumn{7}{c}{ MR } & CT & p-value & r-value & 95\% LA (mean) \\
\hline Left Ventricle & & & & & \\
EDV $\left(\mathrm{ml} / \mathrm{m}^{2}\right)$ & $76 \pm 25$ & $74 \pm 21$ & $>0.05$ & 0.59 & $-47.3 ; 53.9(3.3)$ \\
ESV $\left(\mathrm{ml} / \mathrm{m}^{2}\right)$ & $38 \pm 23$ & $37 \pm 19$ & $>0.05$ & 0.76 & $-66.2 ; 71.4(2.6)$ \\
$\mathrm{SV}\left(\mathrm{ml} / \mathrm{m}^{2}\right)$ & $38 \pm 11$ & $37 \pm 13$ & $>0.05$ & 0.44 & $-57.7 ; 65.6(3.9)$ \\
EF $(\%)$ & $52 \pm 14$ & $52 \pm 14$ & $>0.05$ & 0.73 & $-40.9 ; 42.0(0.6)$ \\
ED wall mass & $59 \pm 18$ & $57 \pm 18$ & $>0.05$ & 0.76 & $-37.9 ; 46.3(4.2)$ \\
$\quad\left(g / \mathrm{m}^{2}\right)$ & & & & & \\
Right Ventricle & & & & & \\
EDV $\left(\mathrm{ml} / \mathrm{m}^{2}\right)$ & $80 \pm 23$ & $84 \pm 25$ & $>0.05$ & 0.58 & $-50.2 ; 39.9(-5.2)$ \\
ESV $\left(\mathrm{ml} / \mathrm{m}^{2}\right)$ & $43 \pm 18$ & $46 \pm 21$ & $>0.05$ & 0.70 & $-65.7 ; 53.3(-6.2)$ \\
SV $\left(\mathrm{ml} / \mathrm{m}^{2}\right)$ & $37 \pm 12$ & $38 \pm 12$ & $>0.05$ & 0.55 & $-58.3 ; 51.8(-3.2)$ \\
EF $(\%)$ & $47 \pm 12$ & $47 \pm 12$ & $>0.05$ & 0.74 & $-38.4 ; 42.2(1.9)$ \\
\hline
\end{tabular}

The Table shows global ventricular parameters (Right and Left Ventricle) calculated with MR and CT. Parameters are expressed as mean \pm SD

Abbreviations: $M R$ Magnetic Resonance, $C T$ Computed Tomography, $E D V$ End Diastolic Volume, ESV End Systolic Volume, SV Stroke Volume, $E F$ Ejection Fraction, ED wall mass End Diastolic wall mass, p-Value Student's paired test, $r$-value Pearson's correlation, 95\% LA Limits of agreement with Bland-Altman analysis (mean in parenthesis)
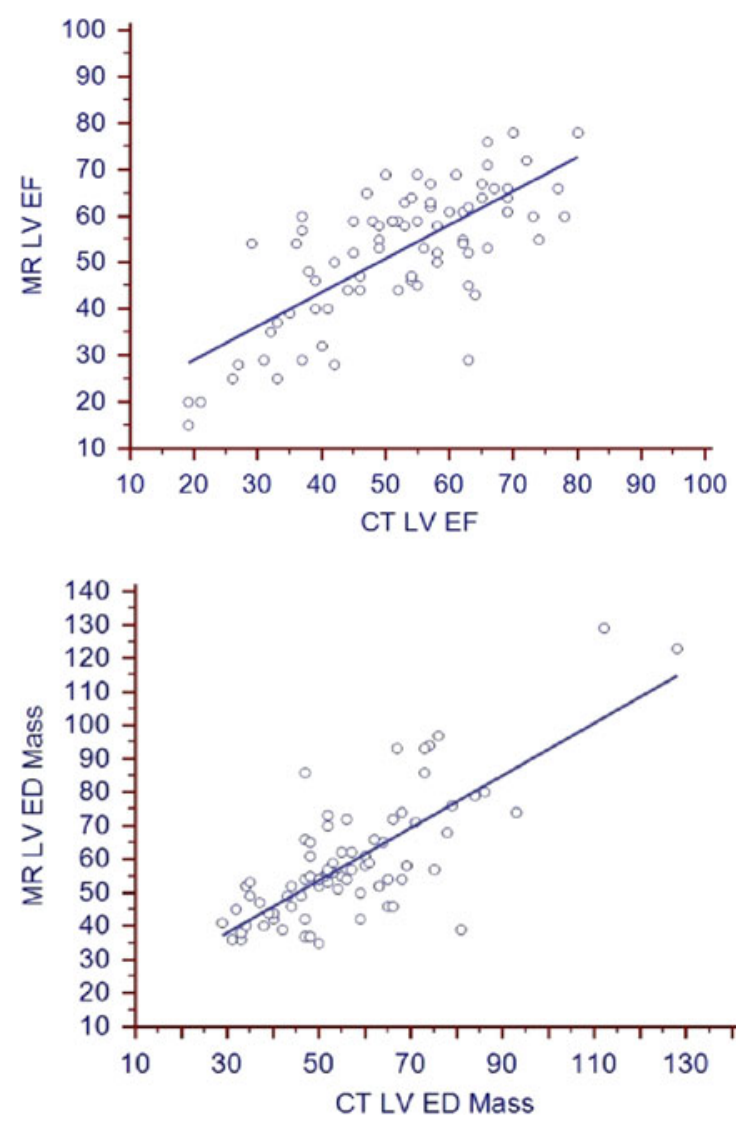

Fig. 3 Scatter plots. Correlation of ejection fraction (EF) values in LV and RV and of ED Mass in LV. The graphs show minimal dispersion of the data and moderate/good correlation $(r>0.7)$ for all displayed right and left ventricular measurements obtained by CT with the results of MR as the currently accepted reference standard for cardiac chamber volume measurement. For data analysis we used commercially available software (MedCalc v9.2.1.0, Mariakerke, Belgium). The correlation between $\mathrm{CT}$ and MR was tested by two-variable linear regression analysis including calculation of Pearson's correlation coefficient. To further examine the agreement between $\mathrm{CT}$ and MRI , Bland-Altman method was used [42] (Fig. 2). The agreement between $\mathrm{CT}$ and MR was determined as the mean difference, the standard error of estimation for the mean difference, the $95 \%$ confidence interval of the mean difference, and the limits of agreement for both investigations (mean \pm SD). Differences were investigated with Student's $\mathrm{T}$ test ( 2 tails) for paired samples and a $p<0.05$ was considered as significant. The comparison of the intra-observer and inter-observer variability between CT and MR was assessed by calculating the coefficient of variability equal to the standard deviation of the difference between two measurements over the mean of the two measurements and expressed as percentage $[16,43]$. Three patient subgroups were created according to $\mathrm{EF}$ value: $<35 \%, 35 \% \leq \mathrm{EF} \leq 50 \%$,

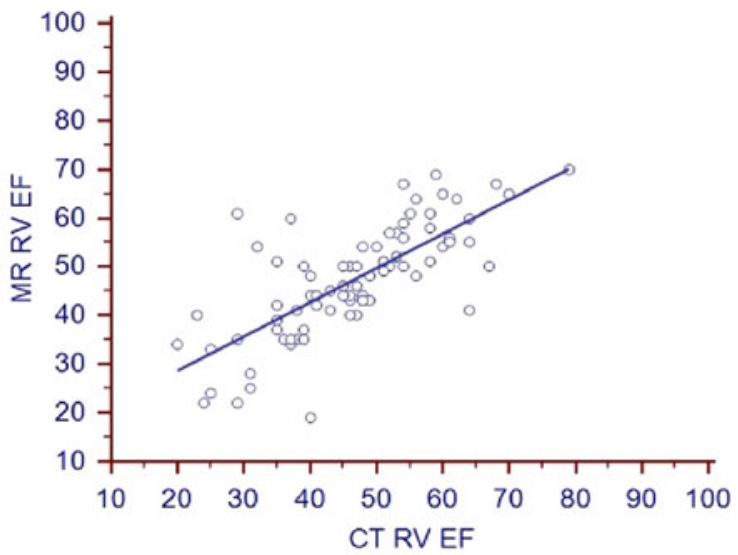

parameters. Abbreviations: EF Ejection Fraction, ED mass End Diastolic wall mass, $M R$ magnetic resonance, $C T$ computed tomography, $S D$ standard deviation 
Table 3 Intra/inter-observer variability

\begin{tabular}{lrrrrrr}
\hline & \multicolumn{3}{c}{$\begin{array}{l}\text { Intra-observer } \\
\text { variability }\end{array}$} & \multicolumn{4}{c}{$\begin{array}{l}\text { Inter-observer } \\
\text { variability }\end{array}$} \\
\hline Left Ventricle & MR & CT & p-value & MR & CT & p-value \\
EDV (\%) & 1.2 & 1.0 & $>0.05$ & 1.3 & 2.3 & $>0.05$ \\
ESV (\%) & 1.8 & 1.3 & $>0.05$ & 2.1 & 3.8 & $>0.05$ \\
SV (\%) & 2.7 & 2.1 & $<0.05$ & 3.4 & 6.4 & $>0.05$ \\
EF (\%) & 2.0 & 1.3 & $>0.05$ & 2.5 & 4.4 & $>0.05$ \\
ED wall mass (\%) & 4.8 & 1.1 & $<0.05$ & 2.9 & 1.6 & $<0.05$ \\
Right Ventricle & & & & & & \\
EDV (\%) & 0.7 & 0.8 & $>0.05$ & 1.7 & 1.0 & $<0.05$ \\
ESV (\%) & 1.2 & 0.9 & $>0.05$ & 2.6 & 1.8 & $>0.05$ \\
SV (\%) & 2.0 & 1.8 & $>0.05$ & 4.3 & 3.1 & $>0.05$ \\
EF (\%) & 1.2 & 1.2 & $>0.05$ & 2.9 & 2.4 & $>0.05$ \\
\hline
\end{tabular}

The Table shows the Coefficient of Variation of ventricular parameters calculated with Deming Regression. Parameters are expressed as percentage variability

Abbreviations: $M R$ Magnetic Resonance, $C T$ Computed Tomography, $E D V$ End Diastolic Volume, ESV End Systolic Volume, SV Stroke Volume, EF Ejection Fraction, ED wall mass End Diastolic wall mass, p-Value Student's paired test

$>50 \%$ and analyzed with Student's T Test, Pearson's correlation and Bland Altman analysis.

\section{Results}

No complications occurred during CT and MR imaging. No patient was excluded due to ECG triggering artifacts. All patients had a regular sinus rhythm with a mean HR of $62 \pm 10 \mathrm{bpm}$ (range: 53-76 bpm) during MSCT and $64 \pm 11 \mathrm{bpm}$ (range: $54-78 \mathrm{bpm}$ ) during MR $(p>0.05)$. The time needed to acquire CT datasets was $12 \pm 3 \mathrm{~s}$ and a complete CT examination took about $5 \pm 3 \mathrm{~min}$; the time needed to acquire MR datasets was $5 \pm 2$ min and a complete MR examination took about $30 \pm 10 \mathrm{~min}(p<0.05)$. LV and RV cavities had good visual quality on all $\mathrm{CT}$ and MR images, with sufficient cavity enhancement. We therefore obtained 79 analyzable datasets for CT and 79 analyzable datasets for MR. Delineation of both left and right ventricular borders took $13 \pm 5 \mathrm{~min}$ for $\mathrm{CT}$ datasets and $25 \pm 7 \mathrm{~min}$ for MR datasets $(p<0.05)$. The mean values and standard deviation for LV and RV volumes and the analysis of the respective differences between $\mathrm{CT}$ and $\mathrm{MR}$ are given in Table 2. Student's paired T test showed no significant differences between $\mathrm{CT}$ and MR for any of the measurements. The limits of agreement between CT and MR were in a good range for all measurements (Fig. 2). Calculation of Pearson's correlation coefficient (r) revealed a good association between $\mathrm{CT}$ and $\mathrm{MR}$ (Fig. 3). The intra-observer and inter-observer variability for both left and right ventricular volumes for CT and MR were calculated with Deming regression and are summarized in Table 3. Reproducibility of CT was significantly higher on an intra-observer level for LV SV and ED Wall Mass, and between readers for the LV ED Wall Mass and RV EDV assessment. Results from the subgroup analysis are listed in Table 4 demonstrating good limits of agreement and an acceptable mean bias for the clinical practice.

\section{Discussion}

Diagnostic and therapeutic decision making in many areas of cardiovascular medicine, including valvular heart disease, ischemic heart disease, ventricular arrhythmias, congenital heart disease, and surgical ventricular restoration planning, requires

Table 4 Functional subgroups and ventricular function parameters

\begin{tabular}{|c|c|c|c|c|c|c|}
\hline Groups & Parameter & MR & $\mathrm{CT}$ & p-value & r-value & 95\% LA (mean) \\
\hline \multirow[t]{2}{*}{ All (n. 79) } & LV EF (\%) & $52 \pm 14$ & $52 \pm 14$ & $>0.05$ & 0.73 & $-40.9 ; 42.0(0.6)$ \\
\hline & RV EF (\%) & $47 \pm 12$ & $47 \pm 12$ & $>0.05$ & 0.74 & $-38.4 ; 42.2(1.9)$ \\
\hline \multirow[t]{2}{*}{$\mathrm{EF}<35 \%$ (n. 11) } & LV EF (\%) & $25 \pm 5$ & $27 \pm 5$ & $>0.05$ & 0.90 & $-22.9 ; 15.6(-3.6)$ \\
\hline & RV EF (\%) & $36 \pm 12$ & $37 \pm 14$ & $>0.05$ & 0.78 & $-61.2 ; 58.1(-1.6)$ \\
\hline \multirow[t]{2}{*}{$35 \% \leq \mathrm{EF} \leq 50 \%$ (n. 18$)$} & LV EF (\%) & $44 \pm 4$ & $46 \pm 10$ & $>0.05$ & 0.52 & $-38.1 ; 30.6(3.8)$ \\
\hline & RV EF (\%) & $44 \pm 9$ & $43 \pm 10$ & $>0.05$ & 0.74 & $-32.9 ; 41.0(4.1)$ \\
\hline \multirow[t]{2}{*}{$\mathrm{EF}>50 \%$ (n. 50$)$} & LV EF (\%) & $61 \pm 7$ & $58 \pm 11$ & $<0.05$ & 0.52 & $-38.1 ; 30.6(-3.8)$ \\
\hline & RV EF (\%) & $51 \pm 10$ & $50 \pm 11$ & $>0.05$ & 0.74 & $-32.9 ; 41.0(4.1)$ \\
\hline
\end{tabular}

The Table shows the comparison of Ejection Fraction (Right and Left Ventricle) calculated with MR and CT for the entire population and for ventricular functional subgroups $(\mathrm{EF}<35 \% ; 35 \% \leq \mathrm{EF} \leq 50 \% ; \mathrm{EF}>50 \%)$. Parameters are expressed as mean $\pm \mathrm{SD}$

Abbreviations: MR Magnetic Resonance, $C T$ Computed Tomography, $n$. number of patients, EF Ejection Fraction, $p$-Value Student's paired test, $r$ value Pearson's correlation, 95\% LA Limits of agreement with Bland-Altman analysis (mean in parenthesis) 
assessment of coronary artery status and concurrent biventricular volumes and function [17]. Currently, MR is considered the standard of reference in LV and RV volume assessment with better accuracy and reproducibility as compared with echocardiography [22]. Recent publications demonstrated excellent agreement between CT and MR in LV and RV volume calculation, and the superiority of CT compared with echocardiography $[14,15,21,44]$. So far, few data are available on concurrent RV and LV volume assessment with $\mathrm{CT}$ as compared with MR. Our results show a good agreement between CT and MR. Only slight differences with no clinical impact were found between analyzed parameters. In particular, LV EDV by CT was lower as compared with MR and RV EDV by CT was higher than MR. This finding further confirm our previous hypothesis that these differences may be caused by different respiratory phases related to different acquisition techniques [14]. The lower temporal resolution of CT is unlikely to be responsible for underestimation of the LV EDV compared with MR, since it is not associated with a consensual overestimation of the LV ESV.

Although we cannot exclude an effect of B-blockers in patients examined with $\mathrm{CT}$, the expected increase in EDV was not observed in our study. Differences may to some extend be explained by the fact that MR images were acquired during expiration, while $\mathrm{CT}$ was performed during an inspiratory breath hold. In maximum inspiration LV venous return is decreased, resulting in EDV reduction and consensual ESV reduction preserving SV and EF. Just the opposite happens for RV venous return, with increased EDV and consensual ESV increment but preserved SV and EF.

Despite good agreement with MR, CT is unlikely to become the preferred imaging investigation for $\mathrm{LV}$ and $\mathrm{RV}$ functional assessment because of the radiation exposure and administration of contrast material. However, for patients with poor echocardiographic compliance, and contra-indications to $\mathrm{MR}, \mathrm{CT}$ can offer a reliable alternative to assess ventricular function. The importance of an alternative functional imaging investigation is even more evident for the RV, which has a complex shape that lends itself poorly for echocardiographic functional assessment. Our results suggest that $\mathrm{CT}$ could be a reliable second option to MR, for instance in patients with (corrected) grown-up congenital heart disease.

The lower intra- and inter-observer variability for LV ED Wall mass by CT may be related to better definition of contours as a result of higher spatial resolution, which improves evaluation of the base slice. Good limits of agreement were maintained after dividing patients in varying EF subgroups, which confirms that cardiac CT can be a reliable tool independently of impaired ventricular function.

CT datasets were analyzed in shorter time compared with MR despite the higher number of images, which may be related to more efficient, semi-automated contours definition on the CT images.
It is important to underline that 21 patients were excluded because of unsuitability for an MR environment, while no patient were excluded because of CT contraindications.

\section{Limitations}

Although the exact radiation dose was not evaluated in this population, it should be mentioned that ECG-gated 64-slice CT without ECG-triggered tube modulation is associated with an exposure between 12-18mSv [45-47]. More modern CT equipment implements different techniques able to acquire datasets useful for coronary tree and LV and RV function evaluation at lower radiation dose [48, 49].

\section{Conclusions}

Using MR as reference, we demonstrated that ECG-gated cardiac $\mathrm{CT}$ accurately assesses both left and right ventricular function, with excellent reproducibility of the functional parameters. Thereby it may be regarded as a reliable alternative to echocardiography and magnetic resonance imaging taking into account the potential risks of radiation and iodinated contrast medium.

Conflict of interest No conflicts of interest to disclose from all Authors. The paper is original and not submitted elsewhere.

Open Access This article is distributed under the terms of the Creative Commons Attribution Noncommercial License which permits any noncommercial use, distribution, and reproduction in any medium, provided the original author(s) and source are credited.

\section{References}

1. Pfeffer MA, Braunwald E, Moyé LA et al (1992) Effect of captopril on mortality and morbidity in patients with left ventricular dysfunction after myocardial infarction: results of the survival and ventricular enlargement trial. N Engl J Med 327:669-677

2. Moise A, Bourassa MG, Theroux P et al (1985) Prognostic significance of progression of coronary artery disease. Am J Cardiol 55:941-946

3. Emond M, Mock MB, Davis KB et al (1994) Long-term survival of medically treated patients in the Coronary Artery Surgery Study (CASS) registry. Circulation 90:2645-2657

4. Juergens KU, Fischbach R (2006) Left ventricular function studied with MDCT. Eur Radiol 16:342-357

5. de Feyter PJ, van Eenige MJ, Dighton DH et al (1982) Prognostic value of exercise testing, coronary angiography and left ventriculography 6-8 weeks after myocardial infarction. Circulation 66:527-536

6. Taylor GJ, Humphries JO, Mellits ED et al (1980) Predictors of clinical course, coronary anatomy and left ventricular function after recovery from acute myocardial infarction. Circulation 62:960-970 
7. White HD, Norris RM, Brown MA et al (1987) Left ventricular end-systolic volume as the major determinant of survival after recovery from myocardial infarction. Circulation 76:44-51

8. Peshock RM, Willett DL, Sayad DE et al (1996) Quantitative MR imaging of the heart. Magn Reson Imaging Clin N Am 4:287-305

9. Rathi VK, Biedermann RW (2004) Imaging of ventricular function by cardiovascular magnetic resonance. Curr Cardiol Rep 6:55-61

10. Alfakih K, Plein S, Thiele H et al (2003) Normal human left and right ventricular dimensions for MRI as assessed by turbo gradient echo and steady-state free precession imaging sequences. J Magn Reson Imaging 17:323-329

11. Thiele H, Nagel E, Paetsch I et al (2001) Functional cardiac MR imaging with steady-state free precession (SSFP) significantly improves endocardial border delineation without contrast agents. J Magn Reson Imaging 14:362-367

12. Barkhausen J, Ruehm SG, Goyen M et al (2001) MR evaluation of ventricular function: true fast imaging with steadystate precession versus fast low-angle shot cine MR imaging: feasibility study. Radiology 219:264-269

13. Moon JCC, Lorenz CH, Francis JM et al (2002) Breath-hold FLASH and FISP cardiovascular MR imaging: left ventricular volume differences and reproducibility. Radiology 223:789797

14. Palumbo A, Maffei E, Martini C et al (2010) Functional parameters of the left ventricle: comparison of cardiac MRI and cardiac CT in a large population. Radiol Med 115:702-713

15. Maffei E, Messalli G, Palumbo A et al (2010) Left ventricular ejection fraction: real-world comparison between cardiac computed tomography and echocardiography in a large population. Radiol Med 115:1015-1027

16. Lembcke A, Dohmen PM, Dewey M et al (2005) Multislice computed tomography for preoperative evaluation of right ventricular volumes and function: comparison with magnetic resonance imaging. Ann Thorac Surg 79:1344-1351

17. Raman SV, Shah M, McCarthy B et al (2006) Multi-detector row cardiac computed tomography accurately quantifies right and left ventricular size and function compared with cardiac magnetic resonance. Am Heart J 151:736-744

18. Belge B, Coche E, Pasquet A et al (2006) Accurate estimation of global and regional cardiac function by retrospectively gated multidetector row computed tomography. Comparison with cine magnetic resonance imaging. Eur Radiol 16:1424-1433

19. Abbara S, Chowa JWB, Pena AJ et al (2008) Assessment of left ventricular function with 16- and 64-slice multi-detector computed tomography. Eur J Radiol 67:481-486

20. Bansal D, Singh RM, Sarkar M et al (2008) Assessment of left ventricular function: comparison of cardiac multidetector-row computed tomography with two-dimension standard echocardiography for assessment of left ventricular function. Int J Cardiovasc Imaging 24:317-325

21. Heuschmid M, Rothfuss JK, Schroeder S et al (2006) Assessment of left ventricular myocardial function using 16-slice multidetector-row computed tomography: comparison with magnetic resonance imaging and echocardiography. Eur Radiol 16:551559

22. Sugeng L, Mor-Avi V, Weinert L et al (2006) Quantitative assessment of left ventricular size and function. side-by-side comparison of real-time three-dimensional echocardiography and computed tomography with magnetic resonance reference. Circulation 114:654-661

23. Wu Y, Tadamura E, Yamamuro M et al (2008) Estimation of global and regional cardiac function using 64-slice computed tomography: a comparison study with echocardiography, gated-SPECT and cardiovascular magnetic resonance. Int J Cardiol 128:69-76

24. Puesken M, Fischbach R, Wenker M et al (2008) Global leftventricular function assessment using dual-source multidetector
CT: effect of improved temporal resolution on ventricular volume measurement. Eur Radiol 18:2087-2094

25. Krishnam MS, Tomasian A, Iv M et al (2008) Left ventricular ejection fraction using 64-slice CT coronary angiography and new evaluation software: initial experience. Br J Radiol 81:450-455

26. Busch S, Johnson TRC, Wintersperger BJ et al (2008) Quantitative assessment of left ventricular function with dual-source CT in comparison to cardiac magnetic resonance imaging: initial findings. Eur Radiol 18:570-575

27. Brodoefel H, Reimann A, Klumpp B et al (2007) Sixty-four-slice $\mathrm{CT}$ in the assessment of global and regional left ventricular function: Comparison with MRI in a porcine model of acute and subacute myocardial infarction. Eur Radiol 17:2948-2956

28. Caudron J, Fares J, Vivier PH et al (2011) Diagnostic accuracy and variability of three semi-quantitative methods for assessing right ventricular systolic function from cardiac MRI in patients with acquired heart disease. Eur Radiol. doi:10.1007/s00330-011-2152-0

29. Guo Y, Gao H, Zhang X et al (2010) Accuracy and reproducibility of assessing right ventricular function with 64-section multidetector row CT Comparison with magnetic resonance imaging. Int J Cardiol 139:254-262

30. Plumhans C, Mühlenbruch G, Rapaee A et al (2008) Assessment of global right ventricular function on 64-MDCT compared with MRI. AJR Am J Roentgenol 190:1358-1361

31. Koch K, Oellig F, Oberholzer K et al (2005) Assessment of right ventricular function by 16-detector-row CT: comparison with magnetic resonance imaging. Eur Radiol 15:312-318

32. Müller M, Teige F, Schnapauff D et al (2009) Evaluation of right ventricular function with multidetector computed tomography: comparison with magnetic resonance imaging and analysis of inter- and intraobserver variability. Eur Radiol 19:278-289

33. Levine GN, Gomes AS, Arai AE et al (2007) Safety of magnetic resonance imaging in patients with cardiovascular devices: an American Heart Association scientific statement from the Committee on Diagnostic and Interventional Cardiac Catheterization. Circulation 116:2878-2891

34. Shellock FG, Spinazzi A (2008) MRI safety update: 2008, Part 2, screening patients for MRI. AJR Am J Roentgenol 191:1221

35. Cademartiri F, Romano M, Seitun S et al (2008) Prevalence and characteristics of coronary artery disease in a population with suspected ischaemic heart disease using CT coronary angiography: correlations with cardiovascular risk factors and clinical presentation. Radiol Med 113:363-372

36. Partridge JB, Anderson RH (2009) Left ventricular anatomy: its nomenclature, segmentation, and planes of imaging. Clin Anat 22:77-84

37. Hergan K, Schuster A, Frühwald J et al (2008) Comparison of left and right ventricular volume measurement using the Simpson's method and the area length method. Eur J Radiol 65:270-278

38. Lorenz CH, Walker ES, Morgan VL et al (1999) Normal human right and left ventricular mass, systolic function, and gender differences by cine magnetic resonance imaging. J Cardiovasc Magn Reson 1:7-21

39. Miller S, Simonetti OP, Carr J et al (2002) MR imaging of the heart with cine true fast imaging with steady-state precession: influence of spatial and temporal resolutions on left ventricular functional parameters. Radiology 223:263-269

40. van Geuns RJM, Baks T, Gronenschild EHBM et al (2006) Automatic quantitative left ventricular analysis of cine MR images by using three-dimensional information for contour detection. Radiology 240:215-221

41. Sievers B, Kirchberg S, Bakan A et al (2004) Impact of papillary muscles in ventricular volume and ejection fraction assessment by 
cardiovascular magnetic resonance. J Cardiovasc Magn Reson $6: 9-16$

42. Bland JM, Altman DG (1986) Statistical methods for assessing agreement between two methods of clinical measurement. Lancet $1: 307-310$

43. Grothues F, Moon JC, Bellenger NG et al (2004) Interstudy reproducibility of right ventricular volumes, function, and mass with cardiovascular magnetic resonance. Am Heart J 147:218223

44. Sugeng L, Mor-Avi V, Weinert L et al (2010) Multimodality comparison of quantitative volumetric analysis of the right ventricle. JACC Cardiovasc Imaging 3:10-18

45. Alkadhi H (2009) Radiation dose of cardiac CT-what is the evidence? Eur Radiol 19:1311-1315
46. Hausleiter J, Meyer T, Hadamitzky M et al (2006) Radiation dose estimates from cardiac multislice computed tomography in daily practice: impact of different scanning protocols on effective dose estimates. Circulation 113:1305-1310

47. Achenbach S, Anders K, Kalender WA (2008) Dual-source cardiac computed tomography: image quality and dose considerations. Eur Radiol 18:1188-1198

48. Hausleiter J, Martinoff S, Hadamitzky M et al (2010) Image quality and radiation exposure with a low tube voltage protocol for coronary CT angiography results of the PROTECTION II Trial. JACC Cardiovasc Imaging 3:1113-1123

49. Alkadhi H, Leschka S (2011) Radiation dose of cardiac computed tomography - what has been achieved and what needs to be done. Eur Radiol 21:505-509 